\title{
Models and Algorithms for Social Distancing in Order to Stop the Spread of COVID-19
}

\author{
Alexandru Popa ${ }^{a b}$
}

\begin{abstract}
Currently there are many attempts around the world to use computers, smartphones, tablets and other electronic devices in order to stop the spread of COVID-19. Most of these attempts focus on collecting information about infected people, in order to help healthy people avoid contact with them. However, social distancing decisions are still taken by the governments empirically. That is, the authorities do not have an automated tool to recommend which decisions to make in order to maximize social distancing and to minimize the impact for the economy.

In this paper we address the aforementioned problem and we design an algorithm that provides social distancing methods (i.e., what schools, shops, factories, etc. to close) that are efficient (i.e., that help reduce the spread of the virus) and have low impact on the economy.

On short: a) we propose several models (i.e., combinatorial optimization problems); b) we show some theoretical results regarding the computational complexity of the formulated problems; c) we give an algorithm for the most complex of the previously formulated problems; d) we implement and test our algorithm.
\end{abstract}

Keywords: combinatorial optimization, COVID-19, algorithm, NP-hard problem

\section{Introduction}

The rapid spread of COVID-19 around the world is stunning. This novel coronavirus created an unprecedented lockdown in many countries which, in turn, caused an immense economic and social impact. Thus, many researchers investigate methods to stop this epidemic as soon as possible. For example, the list of papers on COVID-19 collected by the World Health Organization [28] contains around 10000 publications, a huge number, given that the virus was first discovered in January 2020. The struggle involves researchers from various fields such as bioinformatics, epidemiology, sociology, mathematics and computer science.

\footnotetext{
${ }^{a}$ Department of Computer Science, University of Bucharest, Romania

${ }^{b}$ E-mail: alexandru.popa@fmi.unibuc.ro, ORCID: 0000-0003-3364-1210
} 
One key factor to stop the spread of the virus is the social distancing (see, e.g., [10]). Many companies and organizations try to develop applications to aid the social distancing (see [25] for a long list of current such projects). However, many applications seem to focus on tracking people movement. To the best of our knowledge, we do not know any application that advises the authorities which decisions to make. As Thomas Pueyo writes in his article published on the 19th of March 2020 [20] (Chart 16), governments should have a chart with the effect and the cost of various social distancing measures. As it is currently observed in the world (and especially in Europe), many governments were afraid to take severe social distancing measures in order to avoid a high economic loss.

In this paper we try to address this issue as follows. We first build a model (i.e., a combinatorial optimization problem) that captures the current setting: the risk of the people to get COVID-19, the contact between various people and the cost of closing various facilities such as schools, parks, cities, factories, etc.. We show that the problem we introduce is NP-hard (as it is often the case with complex combinatorial optimization problems). Then, since we cannot solve the problem exactly in polynomial time, we provide a heuristic polynomial time algorithm for this problem. To understand the performance of our algorithm, we implement and test it in Section 5. We generate our test data using special probability distributions that simulate real world social networks as we present in Section 5.1. Our experiments are encouraging and show that even with a $1 \%$ budget (from the total cost of locking down the entire country), we can reduce the population risk by more than 5 times compared with the situation in which no measures are taken. Thus, we show that there is a possibility for a "beautiful" lockdown that is efficient in the fight with COVID-19 and safe for the economy.

\subsection{Related work}

In this paragraph we present briefly the related work in the field. Since the number of papers written on the topic is huge, it is impossible to mention all the results. Nevertheless, we enumerate a couple of papers that we consider relevant to the current work.

We first mention that some of the models presented in the paper are connected to random graphs. Random graphs is an active area of research which combines probability theory and graph theory. The subject began in 1960 with the seminal paper of Erdös and Rényi [6]. The book by Bollobás [5] is the standard source for the field. Other notable sources are $[11,7,15,2]$.

We now briefly mention the role of operations research in developing epidemic response strategies. One of the main applications of operations research was healthcare, e.g. $[21,12,30,24,4,18]$. However, most of the papers in the operations research field are concerned with either simulation frameworks $[27,23,16]$ or resource allocation problems $[22,17,13,3]$.

A few models in the literature attempted to evaluate and devise response strategies among which we mention $[26,14,31]$. For more details, we refer the reader to the survey of Yu et al. [29], the related work section in the paper of Gillis et al. [9], 
and the survey of Adiga et al. [1].

We mention that the paper is written in a bottom-up fashion. More precisely, in Section 2 we present a preliminary model that we designed in the early stages of our study. Even if we do not consider the model in Section 2 further in the paper, the motivation for introducing it is two-fold. Firstly, by presenting the model in Section 2 we show the reader the complete path we took to design the model in Section 3 (instead of simply presenting the final product). Secondly, researchers who aim to study and improve the models presented in this paper may find useful to understand the difficulty behind designing a comprehensive model.

The paper is structured as follows. At first, in Section 2 we present the first set of problems that aim to model the problem. We also show that these problems are NP-hard. Then, in Section 3 we present our actual framework. In Section 4 we design an algorithm for the problem presented in Section 3. Then, in Section 5 we describe our experiments. Finally, in Section 6 we discuss several directions for future work.

\section{Preliminary ideas}

In this section we introduce a preliminary model (i.e., a collection of related combinatorial optimization problems) that helped us to derive the model from Section 3.

\subsection{A tentative framework}

The input consists of an undirected complete graph $G=\left(V,\left(\begin{array}{l}V \\ 2\end{array}\right)\right)$ and a function $p:\left(\begin{array}{c}V \\ 2\end{array}\right) \rightarrow[0,1]$. Each node $v \in V$ in the graph corresponds to a person and $p(u, v)$ is the probability that two people get in contact with each other. Moreover, each vertex $v \in V$ has two associated values, risk $V \rightarrow[0,1]$ and vulnerability $: V \rightarrow$ $[0,1]$, representing how likely is a person to spread the disease (e.g., it can be 1 if a person is tested positive with COVID-19 or close to 1 if a person was recently in a "red area"), respectively how vulnerable is a certain person (e.g., there are studies showing that elderly people and people with chronic diseases are more likely to be affected).

Besides the input graph we are given $k_{1}$ sets of vertices $V^{1}=\left\{V_{1}^{1}, V_{2}^{1}, \ldots, V_{k_{1}}^{1}\right\}$ each one having associated a value $c_{1}:\left\{1,2, \ldots, k_{1}\right\} \rightarrow \mathbb{R}_{+}$and a value $r_{1}$ : $\left\{1,2, \ldots, k_{1}\right\} \rightarrow[0,1]$. The cost $c_{1}(i)$ represents the cost of reducing the value of all $p(a, b), \forall a, b \in V_{i}^{1}$ to $p(a, b) \cdot r_{1}(i)$. Informally, the cost $c_{1}(i)$ represents the cost of closing facility $i$ (i.e., a school, a bar, restaurant, theater, etc.), which in turn reduces the probability of interaction of people belonging to the corresponding facility. In a simple variant, each $r_{1}(i)$ can be set to 0 , representing that two people who belong to that facility will have probability 0 to interact once the facility is closed.

Then, we have $k_{2}$ sets of vertices $V^{2}=\left\{V_{1}^{2}, V_{2}^{2}, \ldots, V_{k_{2}}^{2}\right\}$ each one with a value $c_{2}:\left\{1,2, \ldots, k_{2}\right\} \rightarrow \mathbb{R}_{+}$and a value $r_{2}:\left\{1,2, \ldots, k_{2}\right\} \rightarrow[0,1]$. The cost $c_{2}(i)$ represents the cost of reducing the value of all $p(a, b)$ to $p(a, b) \cdot r_{2}(i)$, where $a \in V_{i}^{2}$ 
and $b \notin V_{i}^{2}$. Informally, the cost $c_{2}(i)$ represents the cost of isolating the people in the group $V_{i}^{2}$ (for example, quarantining persons, small groups or even closing entire cities).

\subsection{Possible combinatorial optimization problems}

Now we introduce a couple of objective functions and constraints that aim to model the current scenario. The overall goal is to reduce the spread of the virus while keeping the cost at a minimum. The first group of problems consider a simplified variant of the framework, ignoring the vulnerability and the risk of each person.

In the first problem the goal is to optimize the economic cost of closing various facilities and isolating various groups of people, while maximizing the number of components created.

Problem 1. We are given a budget $B \in \mathbb{R}_{+}$and a threshold $P \in[0,1]$. The goal is to select a set $\hat{V}^{1} \subseteq V^{1}$ and a set $\hat{V}^{2} \subseteq V^{2}$ such that the following two conditions are met:

1.

$$
\sum_{i \in \hat{V}^{1}} c_{1}(i)+\sum_{i \in \hat{V}^{2}} c_{2}(i) \leq B
$$

2. After the sets of facilities $\hat{V}^{1}$ and $\hat{V}^{2}$ are selected and the corresponding edges have their probabilities decreased (as described in Subsection 2.1), we remove all the edges $(a, b) \in\left(\begin{array}{c}V \\ 2\end{array}\right)$ such that $p(a, b) \leq P$. The goal is to maximize the number of connected components in the remaining graph.

As we stated above, the model does not consider all the information. However, it is useful in cases where not much data is available to conduct preliminary tests. Moreover Problem 1 is interesting to study from the theoretical point of view since it is a novel combinatorial optimization problem.

Notice that even this oversimplified variant of the framework is NP-hard since it is a generalization of the classical Vertex Cover problem as we show in Subsection 2.3 .

The second problem that we introduce is similar to the first problem. Here the goal is to minimize the budget, while requiring for at least a certain number of connected components to be created.

Problem 2. We are given a number of desired connected components $N$ and a threshold $P \in[0,1]$. The goal is to select a set $\hat{V}^{1} \subseteq V^{1}$ and a set $\hat{V}^{2} \subseteq V^{2}$ such that the following holds. After the sets of vertices $\hat{V}^{1}$ and $\hat{V}^{2}$ are selected and the corresponding edges have their probabilities decreased, we remove all the edges $(a, b) \in\left(\begin{array}{c}V \\ 2\end{array}\right)$ such that $p(a, b) \leq P$. The number of connected components in the remaining graph should be at least $N$. The goal is to minimize

$$
\sum_{i \in \hat{V}^{1}} c_{1}(i)+\sum_{i \in \hat{V}^{2}} c_{2}(i)
$$


If we ask to maximize only the number of connected components we might obtain a solution that does not match the original motivation. For example, we can obtain a solution where we have many small components and a huge component, which is, of course, not desired in practice. Thus, we introduce the following two problems, in which we impose a restriction on the size of the connected components resulted after the closure of facilities.

Problem 3. The input is the same as in Problem 1. The goal is to minimize the number of nodes of the largest connected components in the remaining graph.

Problem 4. The input is the same as in Problem 2, but $N$, instead of being the number of connected components desired, is the maximum allowed size of a connected component. Thus, the goal is to choose a set of facilities of minimum total budget (if such a set exists) such that, after closing these facilities, each resulting component has size less than or equal to $N$.

In the end of this section, we formulate two more complex problems that aim to take into considerations all the restrictions, including the risk and the vulnerability.

Problem 5. Besides the input graph and the data associated with the facilities, we are given a budget $B$, a threshold $P$ and two real numbers $W$ and $R$. We have the following constraints associated with the connected components resulted after closing the facilities:

1. For any connected component $X$ we have $\sum_{v \in X}$ vulnerability $(v) \leq W$. Informally, this constraint aims to avoid large groups formed by vulnerable people (such as eldery, or immunosuppressed).

2. For any connected component $X$ we have $\sum_{u, v \in X}(\max \{\operatorname{risk}(u)$, risk $(v)\}-$ $\min \{\operatorname{risk}(u)$, risk $(v)\}) \leq R$. Informally, this constraint aims to avoid a connected component that mixes "healthy" and "ill" people. Notice that if two people have high risk (i.e., that are very likely to have COVID-19) or if two people have very low risk, then $\max \{\operatorname{risk}(u), \operatorname{risk}(v)\}-\min \{\operatorname{risk}(u), \operatorname{risk}(v)\}$ is very close to 0 .

The goal is to select a set of facilities such that, after removing the edges with probability less than $P$, minimises the number of connected components that violate any of the two above mentioned constraints.

The final problem that we propose in this section, is very similar to Problem 5 but aims to enforce that all the components resulted obey the restrictions. Nevertheless, in this variant, we are not given a constraint on the budget. Otherwise, if we are given a constraint on the budget and on the connected components, it is NP-hard even to decide if a feasible solution exists (we obtain an instance of the Knapsack problem that is NP-hard [8]).

Problem 6. The input is similar to Problem 5, except that we do not have a budget $B$. The goal is to select a set of facilities of minimum cost (if such a set exists) such that, after removing the edges with probability less than $P$, all the connected components do not violate any of the two constraints defined in Problem 5. 


\subsection{Hardness results}

In this section we show that the problems introduced in Subsection 2.2 are NPhard. We show a complete proof only for Problem 1, since the NP-hardness proofs for the other problems are similar.

Theorem 1. Problem 1 is NP-hard.

Proof. We show a simple reduction from the Vertex Cover problem which is a classical NP-hard problem [8]. In the (decision version of the) Vertex Cover problem the input is an undirected graph $G=(V, E)$ and an integer $k$ and the goal is to decide, if exists, a subset $V^{\prime} \subseteq V$ such that $\left|V^{\prime}\right| \leq k$ and for any edge $(a, b) \in E$, either $a \in V^{\prime}$ or $b \in V^{\prime}$ or both. Thus, given an instance of Vertex Cover, that is, a graph $G=(V, E)$ and an integer $k$, we construct an instance of Problem 1 as follows.

1. The input graph $G^{\prime}$ of Problem 1 has the same vertex set $V$.

2. The edge set is constructed as follows: for every edge $(a, b) \in E$, we set $p(a, b)=1$, otherwise we set $p(a, b)=0$.

3. We let $V^{1}=\emptyset$.

4. We let $V^{2}=\{\{v\} \mid \forall v \in V\}$, while the cost $c_{2}$ of selecting any set from $V^{2}$ is 1 and $r_{1}$ is 0 (that is, all the edges that are incident to a selected vertex are deleted).

5. The budget $B=k$.

Now, we show that the graph $G=(V, E)$ has a vertex cover of size at most $k$ if and only if the maximum number of connected components in the corresponding instance of Problem 1, after removing the edges $(a, b)$ with $p(a, b)=0$, is $n$.

First, given a vertex cover $V^{\prime}$, the solution of Problem 1 that creates $n$ connected components selects the set $\hat{V}^{2}=\left\{\left\{v^{\prime}\right\} \mid v^{\prime} \in V^{\prime}\right\}$, that is, we select the sets from $V^{2}$ corresponding to the vertices in $V^{\prime}$. Since $V^{\prime}$ is a vertex cover, any edge is incident to at least one vertex from $V^{\prime}$, thus $p(a, b)=0, \forall a, b \in V$ after selecting $\hat{V}^{2}$.

Conversely, given a set $\hat{V}^{2}$, such that $\left|\hat{V}^{2}\right| \leq k$, we construct the set $V^{\prime}=$ $\left\{v^{\prime} \mid\left\{v^{\prime}\right\} \in \hat{V}^{2}\right\}$. Since $n$ connected components are created after selecting $\hat{V}^{2}$, we know that $p(a, b)=0, \forall a, b \in V$ (otherwise, we have a connected component with at least two vertices). Since $p(a, b)=0, \forall a, b \in V$, we know that for any edge $(a, b)$ that had $p(a, b)=1$, either $\{a\} \in \hat{V}^{2}$ or $\{b\} \in \hat{V}^{2}$. Thus, $V^{\prime}=\left\{v^{\prime} \mid\left\{v^{\prime}\right\} \in \hat{V}^{2}\right\}$ is a vertex cover of $G$, completing the proof.

Using a similar reduction, we can show that Problems 2, 3, 4, 5 and 6 are NP-hard. Thus, we state the following corollary.

Corollary 1. Problems 2, 3, 4, 5 and 6 are NP-hard. 


\section{The framework for modeling COVID-19}

The framework presented in the previous section, although promising, has the following problem. The closure of a facility might not have the same effect for all the people that are connected through that facility. Consider the following simple example: two siblings (who live in the same house) study at the same school. Then, after closing the school, in reality the two siblings still have a large probability to get in contact with each other. Thus, we introduce the following framework which captures the aforementioned example and is also simpler than the framework presented in Section 2.

Problem 7. The input consists of a bipartite graph $G=(U \cup V, E)$. The set $U$ represents the people and the set $V$ represents the facilities. For each edge we have associated a value $t: U \times V \rightarrow[0,1]$ that represents the percentage of the time spent by a person in that facility in a day. For example, if $t(a, b)=0.25$, then person a spends 6 hours $(0.25 \times 24$ hours $)$ in facility $b$. Each person has an associated probability $f: U \rightarrow[0,1]$ of being infected. Each facility has an associated closure cost $c: V \rightarrow \mathbb{R}_{+}$. Closing a facility $v$ is equivalent to removing the edges incident to $v$. Moreover, we are given a cost $c^{\prime}: U \rightarrow \mathbb{R}_{+}$of isolating people. Isolating a subset of people $U^{\prime}$ is equivalent to removing the edges incident to all the vertices in $U^{\prime}$. Moreover, we are given a total budget $B$ for closing the facilities.

The risk of a facility is informally the weighted (using the probability of a person being infected $f$ as the weight) sum of the time spent by the people in that facility. More precisely, $R: V \rightarrow \mathbb{R}_{+}$is:

$$
R(v)=\sum_{u \in U:(u, v) \in E} f(u) \cdot t(u, v)
$$

The risk of a person $r: U \rightarrow \mathbb{R}_{+}$(not to be confused with $f$ ) is defined as the weighted sum spent by a person in the facilities he visits (weighted using the risks of the facility). Formally:

$$
r(u)=\sum_{v \in V:(u, v) \in E} R(v) \cdot t(u, v)
$$

We define $r(U)$ the vector in $\mathbb{R}^{|U|}$, that has in each component the risk of a person.

The goal is to select a set of facilities of total cost at most $B$ such that a given function $F: r(U) \rightarrow \mathbb{R}$ is minimized. In this paper we study the case when $F$ is the $\ell_{1}$ metric. In other words, we aim to optimize the total risk of the people.

We show that Problem 7 is NP-hard even in an extremely restricted version in which there is only one person associated with each facility. Nevertheless, notice that unlike Problem 6, Problem 7 admits a trivial feasible solution. Thus, in Section 4 we tackle the problem via a heuristic algorithm and show that it gives promising results. 
Theorem 2. Problem 7 is NP-hard in the case $F=\ell_{1}$.

Proof. We prove NP-hardness of Problem 7 via a reduction from the Subset Sum problem, defined as follows. In the Subset Sum problem the input is a set $S$ of integers and an integer $B$ and the goal is to decide if there exists a subset of integers from $S$ whose sum is precisely $B$. The Subset Sum problem is a famous NP-hard problem [8]. Given an instance of the Subset Sum problem, we create an instance of Problem 7 as follows.

For each $x \in S$, we create a facility $v$ of $\operatorname{cost} c(v)=x$. Thus, $V$ includes these $v$ vertices. The bipartite graph $G=(U \cup V, E)$ with vertex classes $U$ and $V$ is a balanced one, that is, $|U|=|V|$, and the edge set of $G$ is a perfect matching $M=E$. In other words, every vertex of $G$ has degree one.

For every $u \in U$ we let $f(u)=\frac{c(v)}{\max _{w \in V} c(w)}$ and $t(u, v)=1$, where $v$ is the only neighbor of $u$, that is $(u, v) \in M$. Thus, for each pair person/facility $(u, v) \in M$ we have $R(v)=r(u)=f(u)$. Note that if a set of facilities (some subset of $V$ ) is closed with cost $X$, then the total risk is

$$
\frac{\sum_{w \in V} c(w)-X}{\max _{w \in V} c(w)} .
$$

The above shows that the total risk is

$$
\frac{\sum_{w \in V} c(w)-B}{\max _{w \in V} c(w)}
$$

if and only if there exist a subset of numbers from $S$ that have sum precisely $B$.

Thus, Problem 7 is NP-hard in the case $F=\ell_{1}$.

\section{The algorithm}

In this section we provide a heuristic (approximation) algorithm for Problem 7. We test our algorithm in Section 5 and show that it gives promising results.

Our algorithm (presented in Algorithm 1) sorts the list of people and the facilities according to their efficiency (the cost of isolating/closing a person/facility divided by the amount of risk the people/facilities have). Then, the algorithm aims to find the optimum division of the available budget between isolating people and closing facilities. According to our experiments (see Section 5) there is not an obvious correlation between the optimal value of the division of the budget (i.e., variable Split in Algorithm 1) and the minimum total risk. Thus, we need to iterate over all values of Split in order to find a good solution. Of course, since there are infinitely many numbers between 0 and 1 , we cannot iterate over all possible values. Choosing a larger increment improves the running time but reduces the accuracy of the solution. 
1. Define the efficiency of a facility $v$ as

$$
e(v)=\frac{c(v)}{R(v)}
$$

2. Define the efficiency of isolating a person $u$ as

$$
e^{\prime}(u)=\frac{c^{\prime}(u)}{f(u)}
$$

3. Sort the sequence of values $e$ and $e^{\prime}$ in increasing order.

4. MinRisk $=\infty$

5. For every value of Split between 1 and 100 (in increments of 1) do:

a) Isolate people in the order given by $e^{\prime}$ until a budget of $B \cdot \frac{1}{\text { Split }}$ is reached.

b) Close the facilities in the order given by $e$ until the budget $B$ is reached.

c) Let $R_{\text {Split }}$ be the total risk of the population according to this solution. If $R_{\text {Split }}<$ MinRisk then we update the value of MinRisk and store the current solution.

6. Output: MinRisk and the corresponding set of people and facilities that have to be isolated/closed.

Algorithm 1: A heuristic algorithm for Problem 7.

\section{Experiments}

\subsection{Data generation}

In this subsection we describe how we generated our data.

First our data generator allows two parameters as input that determine the number of facilities and the maximum size of a facility. The size of the facilities (i.e., how many people visit that facility in a day) is drawn according to a power law distribution with exponent $\alpha$ (in our experiments $\alpha$ varies between 0.8 and 1.3). We also select an average number of daily activities for a person (i.e., how many facilities a person visits during one day). In our experiments the average number of activities is set between 3 and 8 ). Then, we set the number of people in a country to be the sum of all the facilities divided by the average number of facilities a person visits during one day. 
In Figure 1 we show an example of the distribution of the size of the facilities for 1000 facilities each having a size between 10 and 10000 .

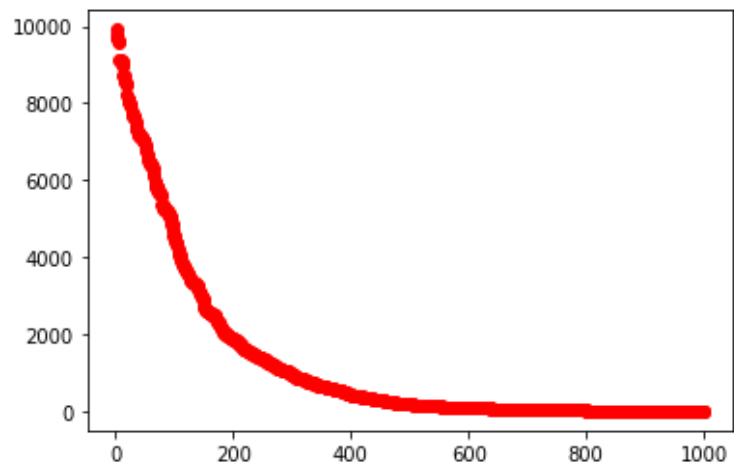

Figure 1: The size of 1000 facilities (i.e. daily number of people that visit that facility). Each facility has at least 10 and at most 10000 daily visitors. The number of visitors is drawn from a power law distribution with $\alpha=1.1$.

For each facility $v$ we select size $(v)$ people that will visit that facility uniformly at random from the population, where size $(v)$ is the size of facility $v$ that was generated previously using the power law distribution. The number of activities performed daily by each person form a Poisson distribution (see Figure 2 for an example).

We now show how we generate the weights on the edges. For each person, we choose the time spent in each facility using an exponential distribution.

The probability that a person $i$ carries the virus, i.e., $f(i)$, is also drawn from a power law distribution with exponent $\alpha_{2}$. One important thing to notice is that

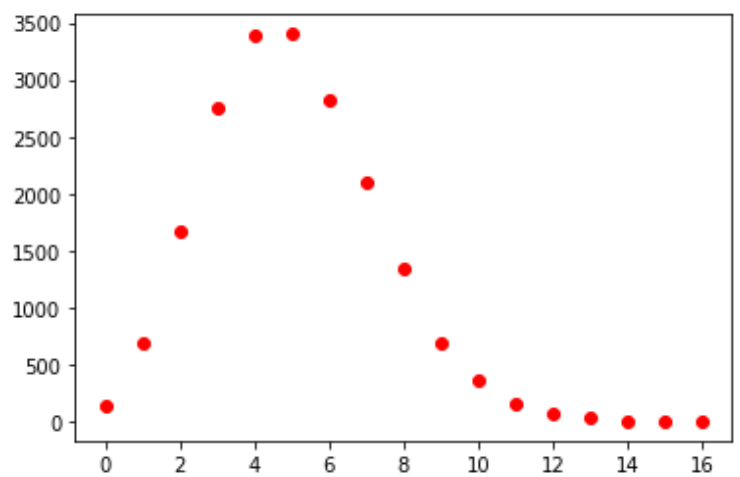

Figure 2: The distribution of number of daily activities for a population of 19573 people that have on average 5 daily activities. 
$\alpha_{2}$ influences significantly the risk of the whole population to get infected. More precisely, if $\alpha_{2}$ is large (that is, there are few people with high risk of carrying the virus), the risk of infection for the other people is relatively low. In Figure 3 we show the risk associated to the people (calculated as shown in Problem 7) for the values of $\alpha_{2}=4$ and $\alpha_{2}=2$. This observation motivates us in the design of algorithm by isolating first the persons with very high risk.

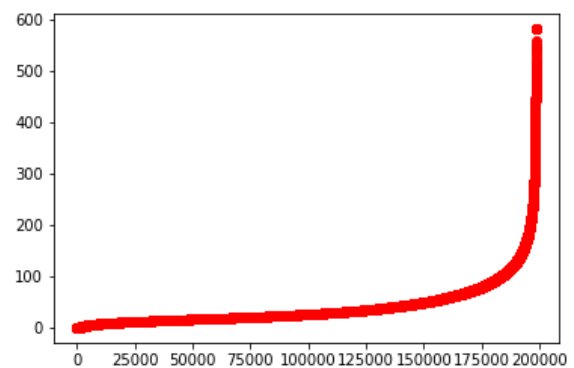

(a) The case of $\alpha_{2}=2$

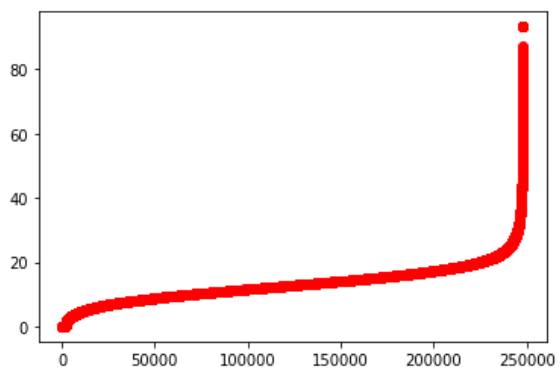

(b) The case of $\alpha_{2}=4$

Figure 3: Comparison of the risk of the population to get infected for $\alpha_{2}=2$ and $\alpha_{2}=4$

Finally, we have to set the cost of isolating people and the cost of closing facilities. We choose the cost of isolating a person as a fraction of total budget available (this fraction can also be set as an input parameter in our generator). The cost of closing a facility of size $s$ is $s^{x}$, where $x$ is a random variable drawn according to a Gaussian distribution with mean $\mu$ and variance $\sigma$ (in our tests we vary the $\mu$ between 1.1 and 1.2 and $\sigma$ between 0.3 and 0.5 ). Finally, the budget is also an input parameter in the generator and we design it as a fraction of the total cost of closing the facilities, generally, between $1 \%$ and $30 \%$.

\subsection{Tests}

We carried out tests for a population of around 30000 people. This population is achieved by varying the parameters in our model as: the number of facilities (between 100 and 1000), the average number of daily activities (between 3 and 8) and the size of each facility (between 4 and 10000). For each set of parameters we carried out 5 tests and we chose the average risk produced by our algorithm over these 5 tests.

The dataset size is the maximum that our hardware can handle. Nevertheless, we argue that our experiments scale to a larger population. In Figure 4 we show how the risk changes if we change the number of facilities and the size of each facility: the risk has a decreasing trend as the size of our population increases, thus we believe that our algorithm is even better for larger scale instances.

Next, we show how the split of the budget between isolating people and closing facilities influences the total risk. In our tests we have 500 facilities between 4 and 
1000 people, each person performs on average 4 activities per day and we have $\alpha=1.1, \alpha_{2}=2$. The cost of the exponent of the random variable that determines the cost of closing the facilities is drawn from a normal distribution with $\mu=1.1$ and $\sigma=0.4$ (Figure 5a and Figure 6a), $\sigma=0.5$ (Figure $5 \mathrm{~b}$ and Figure $6 \mathrm{~b}$ ). The budget is $10 \%$ of the cost of closing all facilities in Figure 5 and $1 \%$ in Figure 6. This budget suffices to isolate $10 \%$ of the population, respectively $1 \%$.

Notice that with a budget of only $1 \%$ from the cost of closing all facilities, we are able to lower the risk to less than $20 \%$ of the original risk (Figure $6 \mathrm{~b}$ ).

Finally, we tested how does the risk decrease if we take actions quickly. More precisely, we vary $\alpha_{2}$ which is the power law exponent that determines the percentage of people that are likely to be already infected. However, we did not notice any major influence of this factor in the total risk if the infection proportion is drawn according to a power law distribution.

Our algorithm was implemented in Python and the tests were carried out on a 2013 MacBook Pro with 2.4 GHz Quad-Core Intel Core i7, and 8GB RAM. The code used for testing and generating data is available on GitHub [19].

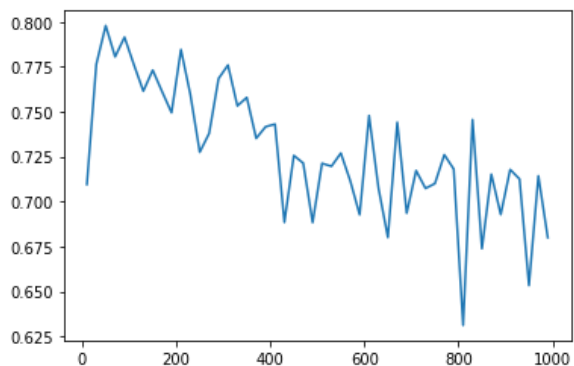

(a) The horizontal axis represents the number of facilities, while the vertical axis represents the risk improvement. The size of each facility is between 4 and 1000 .

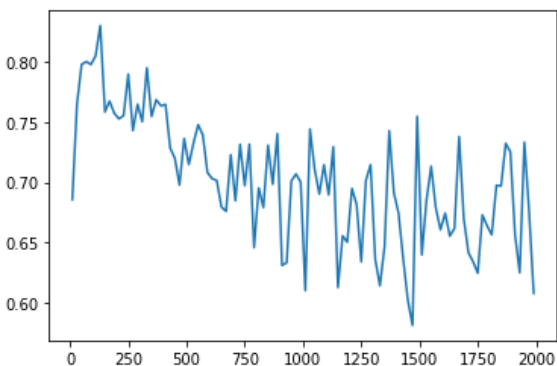

(b) The horizontal axis represents the size of each facility, while the vertical axis represents the risk improvement. The number of facilities is 500 and the average number of daily activities performed by a person is 4 .

Figure 4: The improvement in the population risk (i.e., the risk of the population after our algorithm, divided by the risk before the run of our algorithm) compared with the size of the instance. The average number of daily activities performed by a person is 4 . The budget allocated is $5 \%$ of the amount necessary to close all facilities. With this budget we are able to quarantine $5 \%$ of the population. Then, we have $\alpha=1.1$ and $\alpha_{2}=2$. Observe that the improvement is bigger as the population increases. 


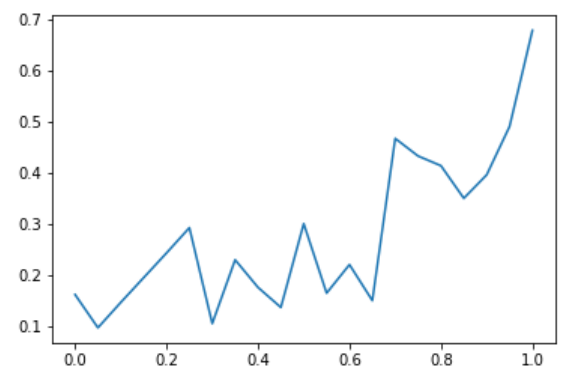

(a) A $10 \%$ of the total cost of closing the facilities and $\sigma=0.4$

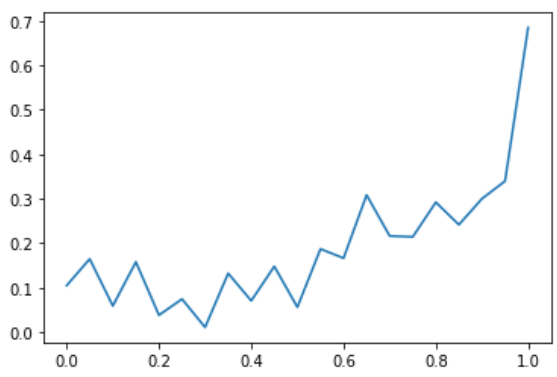

(b) A $10 \%$ of the total cost of closing the facilities and $\sigma=0.5$

Figure 5: On the $x$ axis is the percentage of the total budget allocated to isolating people. On the $y$ axis there is the ratio of the risks before/after running the algorithm.

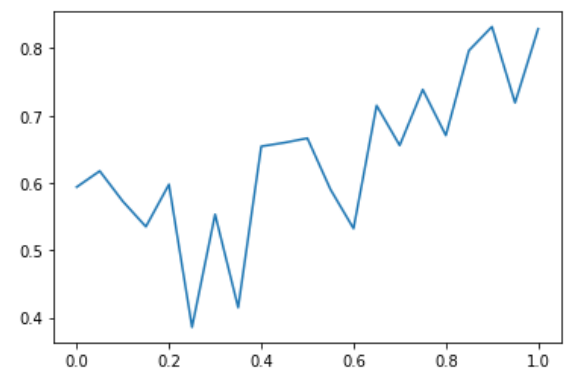

(a) A $1 \%$ of the total cost of closing the facilities and $\sigma=0.4$

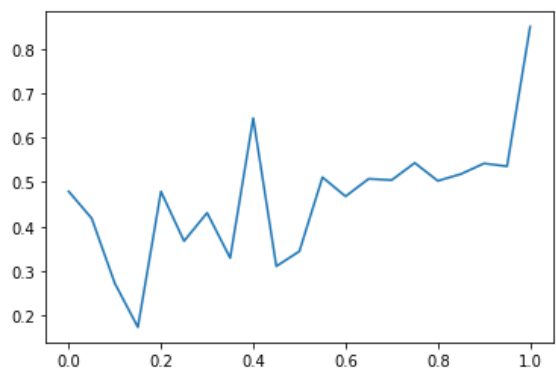

(b) A $1 \%$ of the total cost of closing the facilities and $\sigma=0.5$

Figure 6: On the $x$ axis is the percentage of the total budget allocated to isolating people. On the $y$ axis there is the ratio of the risks before/after running the algorithm.

\section{Conclusions and future work}

In this paper we presented a model and an algorithm that aims to help authorities to take more efficient decisions in the fight with COVID-19. Naturally, the most stringent open problem is to test and validate the model and the algorithm on real data. People have a huge mobility nowadays and it is impossible to create a model which is fully accurate. Nevertheless, based on our tests we believe that our model is capable of capturing the most important features of the current situation. 
Also, a natural open problem is to tune the input parameters: the probabilities $p$ in the input graph, the cost of closing facilities and isolating people $c$ and $c^{\prime}$ and the contagion risk associated with each person.

Since the appearance of vaccines the strategy for tackling COVID has changed significantly. Nevertheless, in some countries, restrictions are still in place. Thus, we are hopeful that our model will give the authorities some insight in taking the best decisions. Moreover, as we can see from our experiments, even with a very small budget (sometimes as low as $1 \%$ of the total cost necessary to lock down the entire economy), the risk of infection can be decreased significantly. Thus, we strongly believe that, with wise decisions, it is possible to stop the spread of COVID-19 and future pandemics without an economic collapse.

\section{Acknowledgements}

I would like to thank Ramona Georgescu, Péter Biró, Radu Mincu and Lucian-Ionut Gavrilă for extremely useful discussions.

\section{References}

[1] Adiga, Aniruddha, Dubhashi, Devdatt, Lewis, Bryan, Marathe, Madhav, Venkatramanan, Srinivasan, and Vullikanti, Anil. Mathematical models for COVID-19 pandemic: A comparative analysis. Journal of the Indian Institute of Science, pages 1-15, 2020. DOI: 10.1007/s41745-020-00200-6.

[2] Alon, Noga and Spencer, Joel H. The Probabilistic Method. Wiley, New York, second edition, 2004. DOI: 10.1002/0471722154.

[3] Bertsimas, Dimitris, Ivanhoe, Joshua Kiefer, Jacquillat, Alexandre, Li, Michael Lingzhi, Previero, Alessandro, Lami, Omar Skali, and Bouardi, Hamza Tazi. Optimizing vaccine allocation to combat the COVID-19 pandemic. medRxiv, 2020. DOI: 10.1101/2020.11.17.20233213.

[4] Biró, Péter, Manlove, David F., and Rizzi, Romeo. Maximum weight cycle packing in directed graphs, with application to kidney exchange programs. Discret. Math. Algorithms Appl., 1(4):499-518, 2009. DOI: 10.1142/ S1793830909000373.

[5] Bollobás, Béla. Modern Graph Theory, volume 184 of Graduate Texts in Mathematics. Springer, 2002. DOI: 10.1007/978-1-4612-0619-4.

[6] Erdos, Paul, Rényi, Alfréd, et al. On the evolution of random graphs. Publ. Math. Inst. Hung. Acad. Sci, 5(1):17-60, 1960.

[7] Frieze, Alan and Karoński, Michał. Introduction to Random Graphs. Cambridge University Press, 2015. DOI: 10.1017/CB09781316339831. 
[8] Garey, M. R. and Johnson, D. S. Computers and Intractability: A Guide to the Theory of NP-Completeness (Series of Books in the Mathematical Sciences). W. H. Freeman, first edition, 1979.

[9] Gillis, Melissa, Urban, Ryley, Saif, Ahmed, Kamal, Noreen, and Murphy, Matthew. A simulation-optimization framework for optimizing response strategies to epidemics. Operations Research Perspectives, 8(C), 2021. DOI: 10.1016/j.orp. 2021.100210 .

[10] Glass, Robert J., Glass, Laura M., Beyeler, Walter E., and Min, H. Jason. Targeted social distancing designs for pandemic influenza. Emerging Infectious Diseases, 12(11):1671, 2006. DOI: 10.3201/eid1211.060255.

[11] Janson, Svante, Luczak, Tomasz, and Rucinski, Andrzej. Random graphs. Wiley-Interscience Series in Discrete Mathematics and Optimization. Wiley, 2000. DOI: $10.1002 / 9781118032718$.

[12] Kahraman, Cengiz and Topcu, Y Ilker. Operations Research Applications in Health Care Management. Springer, 2018. DOI: 10.1007/978-3-319-654553.

[13] Kaplan, Edward H. Containing 2019-nCoV (Wuhan) coronavirus. Health Care Management Science, 23(3):311-314, 2020. DOI: 10.1007/s10729-02009504-6.

[14] Keimer, Alexander and Pflug, Lukas. Modeling infectious diseases using integro-differential equations: Optimal control strategies for policy decisions and applications in COVID-19. Technical Report, 2020.

[15] Kolchin, V. F. Random Graphs. Encyclopedia of Mathematics and its Applications. Cambridge University Press, 1998. DOI: 10.1017/CB09780511721342.

[16] Malone, John D, Brigantic, Robert, Muller, George A, Gadgil, Ashok, Delp, Woody, McMahon, Benjamin H, Lee, Russell, Kulesz, Jim, and Mihelic, F Matthew. US airport entry screening in response to pandemic influenza: Modeling and analysis. Travel Medicine and Infectious Disease, 7(4):181-191, 2009. DOI: $10.1016 / \mathrm{j}$. tmaid.2009.02.006.

[17] Mehrotra, Sanjay, Rahimian, Hamed, Barah, Masoud, Luo, Fengqiao, and Schantz, Karolina. A model of supply-chain decisions for resource sharing with an application to ventilator allocation to combat COVID-19. Naval Research Logistics (NRL), 67(5):303-320, 2020. DOI: 10.1002/nav.21905.

[18] Mincu, Radu Stefan, Biró, Péter, Gyetvai, Márton, Popa, Alexandru, and Verma, Utkarsh. IP solutions for international kidney exchange programmes. Central Eur. J. Oper. Res., 29(2):403-423, 2021. DOI: 10.1007/s10100-02000706-5. 
[19] Popa, Alexandru. A decision support system for optimizing the cost of social distancing in order to stop the spread of COVID-19. https://github.com/ alexpopa9/ResearchCode/blob/master/COVID-19. py, 2020.

[20] Pueyo, Thomas. Coronavirus: The hammer and the dance. https: //medium.com/@tomaspueyo/coronavirus-the-hammer-and-the-dancebe9337092b56, 2020. [Online; accessed 06-04-2020].

[21] Rais, Abdur and Viana, Ana. Operations research in healthcare: A survey. International Transactions in Operational Research, 18(1):1-31, 2011. DOI: 10.1111/j.1475-3995.2010.00767.x.

[22] Risanger, Simon, Singh, Bismark, Morton, David, and Meyers, Lauren Ancel. Selecting pharmacies for COVID-19 testing to ensure access. Health Care Management Science, pages 1-9, 2021. DOI: 10.1007/s10729-020-09538-w.

[23] Roche, Benjamin, Drake, John M, and Rohani, Pejman. An agent-based model to study the epidemiological and evolutionary dynamics of influenza viruses. BMC Bioinformatics, 12(1):1-10, 2011. DOI: 10.1186/1471-2105-12-87.

[24] Roth, Alvin E., Sönmez, Tayfun, and Ünver, M. Utku. Kidney Exchange. The Quarterly Journal of Economics, 119(2):457-488, 05 2004. DOI: 10.1162/ 0033553041382157.

[25] The Governance Lab. Data collaborative in response to COVID19. https://docs.google.com/document/d/1JWeD1AaIGKMPry_ EN8GjIqwX4J4KLQIAqP09exZ-ENI/edit\#, 2020. [Online; accessed 06-042020].

[26] Toda, Alexis Akira. Susceptible-Infected-Recovered (SIR) dynamics of COVID-19 and economic impact. arXiv preprint arXiv:2003.11221, 2020.

[27] Wang, Weixing, Li, Yanli, and Zhang, Jinjin. System dynamics modeling of SARS transmission - a case study of Hebei Province. In 2009 International Conference on Management and Service Science, pages 1-4, 2009. DOI: 10. 1109/ICMSS. 2009.5303731.

[28] World Health Organization. Global research on coronavirus disease (COVID19). https://www. who.int/emergencies/diseases/novel-coronavirus2019/global-research-on-novel-coronavirus-2019-ncov, 2020. [Online; accessed 28-03-2020].

[29] Yu, Shuo, Qing, Qing, Zhang, Chen, Shehzad, Ahsan, Oatley, Giles, and Xia, Feng. Data-driven decision-making in COVID-19 response: A survey. IEEE Transactions on Computational Social Systems, 8(4):1016-1029, 2021. DOI: 10.1109/TCSS. 2021.3075955.

[30] Zaric, Gregory S. Operations Research and Health Care Policy. Springer, 2013. DOI: $10.1007 / 978-1-4614-6507-2$. 
[31] Zhigljavsky, Anatoly, Fesenko, Ivan, Wynn, Henry, Whitaker, Roger, Kremnizer, Kobi, Noonan, Jack, and Gillard, Jonathan. A prototype for decision support tool to help decision-makers with the strategy of handling the COVID19 UK epidemic. medRxiv, 2020. DOI: 10.1101/2020.04.24.20077818.

Received 20th January 2021 